\title{
Treatment of ventriculoperitoneal shunt infection and ventriculitis caused by Acinetobacter baumannii: a case report
}

\author{
Gebre Teklemariam Demoz ${ }^{1 *}$, Minyahil Alebachew², Yirga Legesse ${ }^{3}$ and Belete Ayalneh²
}

\begin{abstract}
Background: Acinetobacter baumannii (A. baumannii) infections are a recognized problem in healthcare, causing ventriculoperitoneal shunt infection and ventriculitis. Such infections are serious intracranial infection that can lead to serious complication and death. Treatment of infection caused by A. baumannii becomes difficult because of its inclination to develop pandrug resistance to the universally used antibiotics. In this case, we focused on pediatric ventriculitis/shunt infection caused by A. baumannii in an extensive follow-up and report the subsequent treatment outcome. Very limited information regarding the therapeutic options against $A$. baumannii ventriculitis/shunt infection is available in our hospital. Thus, we present one such case and the problems in its treatment.

Case presentation: We reported the case of a 6-year-old Ethiopian boy who developed ventriculitis/shunt infection from the pandrug-resistant strain of A. baumannii, after decompression of a craniotomy for medulloblastoma. Following the surgical procedure, he had developed hydrocephalus and ventriculoperitoneal shunt infection/ventriculitis as he presented with persistent fever, elevated white blood cell count, reduced glucose level, and the cerebrospinal fluid culture revealed A. baumannii, which was not responding to most of commercially available antibiotics systemically. Our patient was successfully treated with intravenous ampicillin-sulbactam.
\end{abstract}

Conclusions: We presented our case of pandrug-resistant A. baumannii ventriculoperitoneal shunt infection and ventriculitis successfully treated with a systemic ampicillin-sulbactam. Provision of systemic ampicillin-sulbactam should not be undermined. Therefore, this case exemplifies that intravenous administration of ampicillin-sulbactam can be a good therapeutic option against $A$. baumannii ventriculoperitoneal shunt infection and ventriculitis.

Keywords: Acinetobacter baumannii, Ventriculitis, Hydrocephalus, Ventriculoperitoneal shunt, Extra ventricular drainage (EVD), Multidrug resistance (MDR), Pandrug-resistant (PDR)

\section{Background}

Cerebrospinal fluid (CSF) shunt devices are commonly indicated for the treatment of hydrocephalus in children, and have served to improve survival and reduce mortality. However, ventriculoperitoneal shunt infection is a serious complication, commonly with an underlying bacterial etiology $[1,2]$. Acinetobacter baumannii (A. baumannii) infections are a recognized problem in healthcare, causing ventriculoperitoneal shunt infection and ventriculitis. The incidence of $A$. baumannii resistance is increasing across the globe, and that makes it difficult to treat in

\footnotetext{
* Correspondence: demozgebre5@gmail.com

${ }^{1}$ Clinical Pharmacy and Pharmacy Practice Unit, Department of Pharmacy,

College of Health Sciences, Aksum University, Aksum, Ethiopia

Full list of author information is available at the end of the article
}

neurosurgical practice. It can cause neurological defects, and can lead to a long course of treatment and hospitalization. Such infections are serious intracranial infection that can lead to death $[3,4]$.

Treatment of ventriculoperitoneal shunt infected with A. baumannii is curious because of its inclination to develop pandrug resistance (PDR) to commonly used potent antibiotics in healthcare situations. PDR is defined as when an isolate exhibits resistance to all seven antipseudomonal antimicrobial agents; included antipseudomonal penicillins, cephalosporins, carbapenems, monobactams, quinolones, aminoglycosides, and polymyxins [5]. The Infectious Diseases Society of America (IDSA) recommends vancomycin plus an antipseudomonal $\beta$-lactam (such as cefepime, ceftazidime,

(c) The Author(s). 2018 Open Access This article is distributed under the terms of the Creative Commons Attribution 4.0 International License (http://creativecommons.org/licenses/by/4.0/), which permits unrestricted use, distribution, and 
or meropenem) as empiric therapy for healthcareassociated ventriculitis. For treatment of infection caused by $A$. baumannii, meropenem is strongly recommended; for strains that demonstrate carbapenem resistance, intravenous and/or intraventricular colistin is recommended [6]. In $A$. baumannii ventriculitis, the major problems provoking clinicians in intensive care units are related to the severity of the bacteria and its extraordinary ability to develop multiple resistance mechanisms against major classes of available antibiotics [7].

Little is known regarding the appropriate treatment of ventriculoperitoneal shunt infection and ventriculitis in pediatric patients. Hence, early diagnosis and effective treatment is compulsory. Our primary purpose was to report the successful treatment of ventriculoperitoneal shunt infection and ventriculitis caused by $A$. baumannii with ampicillin-sulbactam. Therefore, this case may be helpful in reminding clinicians to consider ampicillinsulbactam treatment in the management of cases infected with A. baumannii. Thus, this case could also contribute important information for physicians facing such complicated infections.

\section{Case presentation}

A 6-year-old Ethiopian boy was admitted to the pediatric intensive care unit of the Black Lion Specialized Hospital with medulloblastoma and hydrocephalus. He was the first child in his family and came from low socioeconomic status. No history of birth defect, head injury, trauma, central nervous system infections, malignancy and related conditions, except that a year prior, he had been admitted in the private hospital intensive care unit, for more than a month; a ventriculoperitoneal shunt was placed for treating hydrocephalus but there was no recorded history of infections and complications. He presented in our hospital with high-grade global type of headache, poor appetite, nausea, vomiting, tiredness, decreased vision, and drowsiness. These are typical signs and symptoms for medulloblastoma. However, at admission he had no fever, cough, or chest pain. He was also conscious, with a Glasgow Coma Scale (GCS) of 15/15, with no motor deficit or sensory disorder. On admission, his blood pressure (BP) was 140/85 $\mathrm{mmHg}$, with a regular heartbeat of 60 beats per minute, heart sounds were clearly auscultated with no murmur, there was no murmur of mitral insufficiency or ventricular defect, no signs of heart failure, and he was without edema of his lower limbs. His peripheral pulses were perceived symmetrically. The rest of the physical examination was also normal.

On admission, a brain computed tomography (CT) scan and brain magnetic resonance image (MRI) was performed and indicated the presence of medulloblastoma (a mass that was involved the posterior fossa, Fig. 1). An immediate craniotomy procedure was performed for the removal of

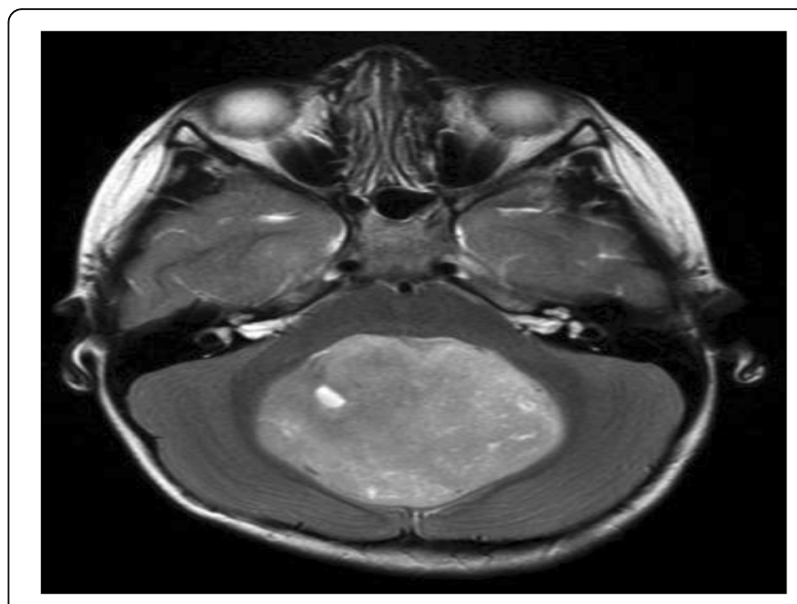

Fig. 1 Contrast magnetic resonance imaging revealing an enhancing posterior fossa mass, involving the third ventricle: medulloblastoma

the mass. A biopsy was also performed and revealed no malignancy or spread of brain tumor. The radiologist and neurologist team suggested that no further treatment was needed, radiotherapy or chemotherapy. However, following the craniotomy procedure, the neurological findings progressively deteriorated. This showed he had developed obstructive hydrocephalus as he presented with gait disturbance, low attention, seizure, and uncontrolled body movement with an elevated intracranial pressure (ICP). To restore this CSF circulation within both lateral ventricles, a ventriculoperitoneal (VP) shunt was placed. However, his mental status could not be improved. Thus, on the 4th day, the VP shunt was replaced with external ventricular drainage (EVD) which was undertaken to control the elevated ICP because of hydrocephalus.

On the 6th of the surgery, he became comatose with a GCS score of 7/15 and hemiparesis. He needed nasogastric tube (NGT) feeding for nutrition. Meanwhile, he presented with high-grade fever $\left(39.8{ }^{\circ} \mathrm{C}\right)$, severe headache, vomiting, lethargy, and deteriorating consciousness. The CSF examination revealed an elevated white blood cell (WBC) count of $21.0\left(10^{9}\right)$ cells/L), reduced blood sugar $(29 \mathrm{mg} / \mathrm{dL})$, and elevated protein level (219 mg/dL).

A blood culture specimen was drawn from his CSF. Awaiting the culture results, an empiric antibiotic therapy was initiated with an intravenous dose of vancomycin and piperacillin-tazobactam. However, he did not respond to this regimen. The second (a day after) CSF result showed that a glucose concentration of $32 \mathrm{mg} / \mathrm{dL}$, a protein level of $260 \mathrm{mg} / \mathrm{dL}$, and a WBC cell count of $20.0\left(10^{9}\right)$ cells/L). The gram staining of the CSF had also shown no organisms. Seven days after surgery, blood culture revealed no growth. 
However, the clinical condition of the patient still did not improve. The vancomycin and piperacillin-tazobactam regimen was changed to cefepime and gentamycin for 7 days. Later, those regimens were also switched to intravenous ciprofloxacin and metronidazole. Despite taking these medications; his fever still persisted and a poor clinical response was observed. Thus, the CSF analysis was repeated on the 18th day of illness and revealed a glucose concentration of $21 \mathrm{mg} / \mathrm{dL}$, a protein level of $200 \mathrm{mg} / \mathrm{dL}$, and a total WBC cell count of $24.0\left(10^{9}\right)$ cells/L). The CSF smear for Gram statin did not reveal any bacteria. However, the patient still had fever; low consciousness level, a low glucose level, and an elevated protein level were noted.

On the 25th day of admission, along with the ventriculoperitoneal shunt infection the MRI showed evidence for ventriculitis, including intraventricular debris in the ventricles on diffusion-weighted imaging and abnormal periventricular intensities on fluid-attenuated inversion recovery imaging (Fig. 2a). Examination of his CSF revealed a persistent elevation of his white blood cell count and reduction in glucose level. This was confirmed with one CSF culture along with a positive Gram stain, which was generated by A. baumannii. Although the shunt system was removed immediately and antibiotic treatment was initiated, the infection persisted despite intravenous administration of antibiotics including vancomycin and piperacillin-tazobactam, cefepime with gentamycin, followed by ciprofloxacin with metronidazole. The bacteria was resistant to all the antibiotics examined in the laboratory by disk diffusion susceptibility test, including $\beta$-lactamase inhibitor penicillins, carbapenems, cephalosporins, fluoroquinolones, and aminoglycosides. However, sensitivity to colistin, aztreonam and tigecycline was not tested because of it was unavailable in our hospital.

Later, with the organism identified; the intravenous antibiotic therapy was continued with ampicillin-sulbactam 1 . $3 \mathrm{~g}$ every $6 \mathrm{~h}$. For CSF isolates of A. baumannii, the minimum inhibitory concentration (MIC) of ampicillinsulbactam was $4 \mu \mathrm{g} / \mathrm{mL}$ (details of each antibiotic MIC is stated in Additional file 1: Tables S1 and S2). Our patient's fever begun to subside and he became afebrile on the 3rd day of the systemic therapy with ampicillin-sulbactam. The CSF culture was performed and was negative on the 10th day of this systemic therapy, and the CSF cell count had reduced to $15.0\left(10^{9}\right)$ cells/L). On the 14th day of the therapy with ampicillin-sulbactam, the CSF culture was completely sterile, the CSF cell count had also dropped to $9.0\left(10^{9}\right)$ cells/L), and the therapy was discontinued. The gradual improvement and clinical outcome of our patient
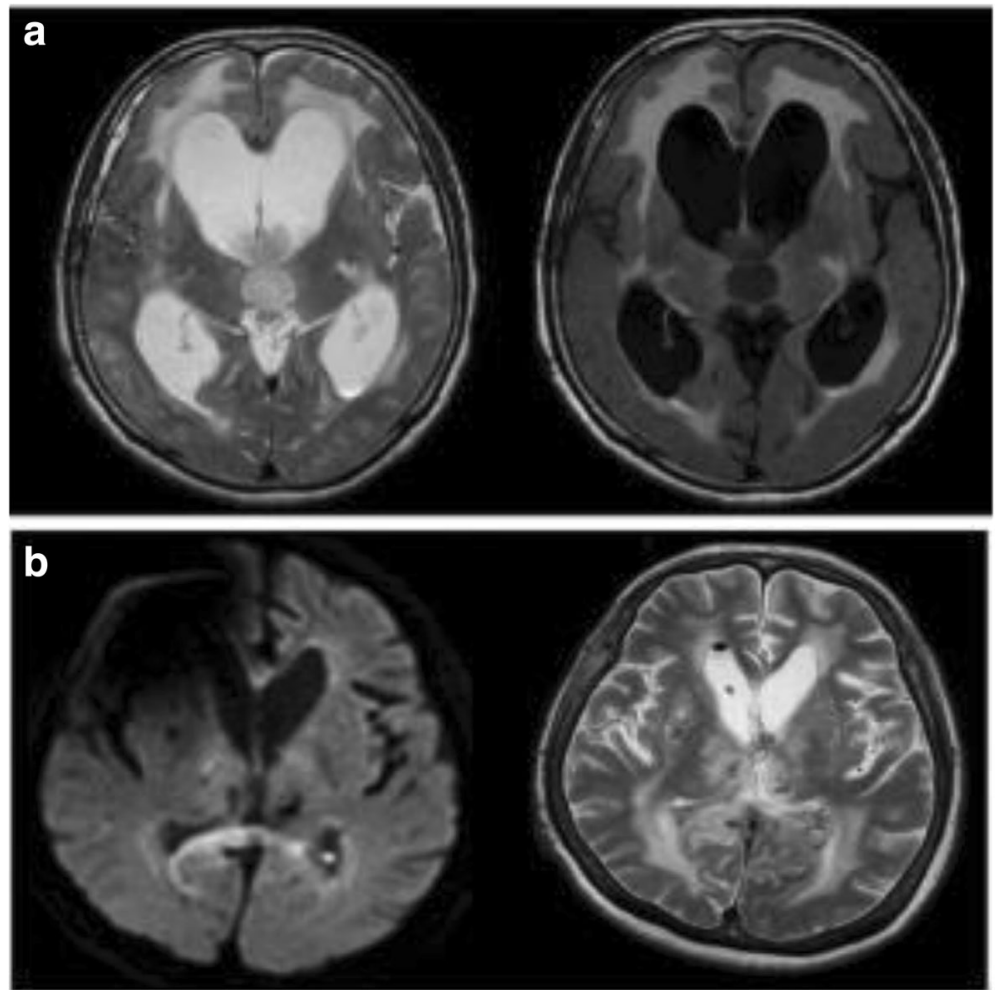

Fig. 2 a Magnetic resonance imaging on the 25th day of admission shows abnormal hyperintensities in the trigones of both lateral ventricles, suggesting debris (left panel). The central panel and fluid-attenuated inversion recovery imaging (right panel) show abnormal periventricular intensities. b During discharge magnetic resonance imaging. Both ventriculitis and hydrocephalus have been resolved 
was noted. Our patient's level of consciousness rapidly improved. The overall condition of the child was improved oddly and progressively, he began to feed orally and became more collaborative. Three consecutive blood culture analyses were performed and revealed no microbial growth all over. Indicating that the infection had gradually subsided and had been cured.

Furthermore, no complications or side effects were witnessed during the treatment. The clinical status of our patient was progressively and remarkably improved. Meantime, after a month the surgical team had diverted the EVD to the left side. Finally, our patient was discharged from the hospital in a stable and good condition. Our patient has been followed up for 2 months after being discharged from the pediatric ward as ambulatory. We found no evidence of recurrence of infection or any neurological defects or malfunction had occurred as of 6 months postoperatively (Fig. 2b). The patient is now able to walk without mobility aids, eat meals by himself, and live independently at home.

\section{Discussion}

We reported the clinical feature and treatment outcome of a single case of a patient with A. baumannii infection. Our case illustrated that the separation of a pandrugresistant mostly for the Gram-negative clinical specimens does not necessarily mean a depraved outcome. This may be clarified by numerous reasons. First, the achieved concentration of several antimicrobial agents in some body fluids, including urine may exceed the minimal inhibitory concentration of the isolated pathogen. Second, infections, even severe ones, are sometimes are self-limiting without the use of antimicrobial agents, as the preantibiotic era taught us [8]. Third, pandrugresistant bacteria may be immigrants in patients receiving several classes of antimicrobial agents for a long period of time, while the real pathogen may not be isolated. Fourth, it has been shown that occasionally multi drug resistance (MDR) organisms may exhibit decreased virulence compared to other more sensitive organisms of the same species [9].

In the management of resistant Gram-negative bacterial infections, combination of colistin with $\beta$-lactam antibiotics, may be a useful agent [5]. Even though, there are already clinical isolates of Gram-negative bacteria that are resistant to all available antibiotics, infections due to resistant Gram-negative bacteria continue to increase. This led to looking at other therapeutic options for these infections, such as colistin and ampicillin-sulbactam combination [10]. However, colistin is not commercially available in our hospital and this led us to use such many of the antibiotics systemically. Finally, we were compelled to use beta-lactam alone. But the treatment outcome of our case was found to be successful. Thus, our case was found to be in line with cases reported of six patients, who were infected with $A$. baumannii meningitis, which were effectively cured with ampicillinsulbactam [11].

One study also reported that the alternative choice drug for the treatment of A. baumannii infections resistant to carbapenems strains were parenteral co-administration of $\beta$-lactam ampicillin with the $\beta$-lactamase inhibitor sulbactam, (ampicillin-sulbactam) [12]. Of the $\beta$-lactamase inhibitors, sulbactam possesses the greatest intrinsic bactericidal activity against $A$. baumannii isolates [13]. The prevalence of drug-resistant strains is increasing, and treatment options are increasingly limited. Effective therapy remains likely when the organism is proven to be susceptible. Thus, a single testing method may result in incorrect susceptibility results and lead the clinician to select a potentially ineffective agent. The challenge for the clinician is to combine local susceptibility patterns with the agents that are most likely to be effective. The use of combination therapy, whether empirical or targeted, has yet to be demonstrated [10]. Despite the partiality for treatment of ventriculoperitoneal shunt infections with antibiotics alone, this mode of treatment was usually not successful; survivors had continued or recurrent infection. Thus, patients with ventriculoperitoneal shunts were treated more frequently by early removal of the shunt followed by administration of systemic and/or local antibiotics [1].

A. baumannii remains an important and difficult-totreat pathogen whose resistance patterns result in significant challenges for the clinician. Despite the prevalence and interest in A. baumannii infections, there is relatively limited well-controlled scientific data to help the clinician in selecting the optimal empirical and subsequent targeted therapy for a variety of infections. Indeed, the pharmacotherapeutic effect of such microorganisms may also need a special attention in neurosurgical cases, while the clinician's proficiency is required to review them based on local and clinical practice.

Numerous studies reported that A. baumannii were multidrug-resistant, which makes it challenging to cure using commercially available potent antibiotics. However, we treated our patient successfully with ampicillinsulbactam intravenously. There were neither recurrences nor superinfections. Thus, eradication of A. baumannii from the CSF in shunt-associated ventriculitis required a complete removal of ventricular devices followed by administration of bactericidal antibiotics.

According to IDSA recommendation, patients with ventriculitis should be monitored for response to treatment based on clinical parameters. For those patients with ventriculitis and an external drainage device, monitoring of CSF cultures is also recommended to ensure that they become negative [14]. In our case, multiple 
monitoring parameters were performed in order to confirm our patient response to the current treatment modalities. In addition, additional CSF analysis was performed to ensure that the CSF parameters had improved and the cultures revealed negative results.

Therefore, we recommend that intravenous combination of ampicillin-sulbactam can be used in such severe and life-threatening infections caused by $A$. baumannii resistance in which there are no available other therapeutic options. This indicates that ampicillin-sulbactam is a good and safe therapeutic option to treat ventriculoperitoneal shunt infection and ventriculitis caused by multidrugresistant $A$. baumannii. We observed that administration of systemic ampicillin-sulbactam therapy for hydrocephalus shunt infection and ventriculitis caused by $A$. baumannii may be effective in reducing the rate of further neurological complication and/or defects and the duration of hospitalization. Moreover, we hope future case studies will explicate a number of definitive and practical options.

\section{Conclusion}

We reported the outcome of a single case of a patient with A. baumannii infection treated with ampicillin-sulbactam and our difficulties in patient management. This drug could be a treatment option against $A$. baumannii when colistin is not available. In our practice, we successfully treated a patient with pandrug-resistant $A$. baumannii ventriculoperitoneal shunt infection and ventriculitis with a systemic ampicillin-sulbactam. As a conclusion, an intravenous administration of ampicillin-sulbactam could be an effective therapeutic option in the treatment of ventriculoperitoneal shunt infection and ventriculitis caused by A. baumannii resistance to most potent antimicrobials, including carbapenem and other $\beta$-lactam drugs. Therefore, even though $A$. baumannii is resistant to most other potent antimicrobials, systemic provision of ampicillinsulbactam should not be undermined.

\section{Additional file}

Additional file 1: Table S1. Medical conditions and pattern of interventions and prescribed antibiotics. Table S2. Sensitivity tests performed and results. (DOCX $21 \mathrm{~kb}$ )

\section{Acknowledgements}

We acknowledge Addis Ababa University, and Black Lion Specialized Hospital healthcare providers for their extensive supervision and follow-up of the patient.

\section{Availability of data and materials}

All data and materials are accessible at any time upon request.

\section{Authors' contributions}

GTD contributed to data collecting, summarized the clinical data, followed the clinical outcome of the patient throughout his stay, and designed and prepared the manuscript. BA, YL, and MA extensively supervised the overall treatment modalities and reviewed and commented the final manuscript. All authors read and approved the final manuscript.
Ethics approval and consent to participate

Not applicable.

\section{Consent for publication}

Written informed consent was obtained from the patient's legal guardian(s) for publication of this case report and any accompanying images. A copy of the written consent is available for review by the Editor-in-Chief of this journal.

\section{Competing interests}

The authors declare that they have no competing interests.

\section{Publisher's Note}

Springer Nature remains neutral with regard to jurisdictional claims in published maps and institutional affiliations.

\section{Author details}

${ }^{1}$ Clinical Pharmacy and Pharmacy Practice Unit, Department of Pharmacy, College of Health Sciences, Aksum University, Aksum, Ethiopia. ${ }^{2}$ Department of Pharmacology and Clinical Pharmacy, School of Pharmacy, College of Health Sciences, Addis Ababa University, Addis Ababa, Ethiopia. ${ }^{3}$ Department of Clinical Pharmacy, School of Pharmacy, College of Health Sciences, Mekelle University, Mekelle, Ethiopia.

Received: 26 June 2017 Accepted: 3 April 2018

Published online: 23 May 2018

References

1. Schoenbaum SC, Gardner P, Shillito J. Infections of cerebrospinal fluid shunts: epidemiology, clinical manifestations, and therapy. J Infect Dis. 1975;131(5):543-52.

2. McClelland S III, Hall WA. Postoperative central nervous system infection: incidence and associated factors in 2111 neurosurgical procedures. Clin Infect Dis. 2007:45(1):55-9.

3. Pelegrin I, et al. Management of ventriculoperitoneal shunt infections in adults: analysis of risk factors associated with treatment failure. Clin Infect Dis. 2017;64(8):989-97.

4. Kadowaki STM. Neuroendoscopic surgery for ventriculitis and hydrocephalus after shunt infection and malfunction: preliminary report of a new strategy. Asian J Endosc Surg. 2015;8(2):180-4.

5. Falagas ME, Bliziotis IA, Kasiakou SK, Samonis G, Athanassopoulou P, Michalopoulos A. Outcome of infections due to pandrug-resistant (PDR) Gram-negative bacteria. BMC Infect Dis. 2005;5:24. https://doi.org/10.1186/ 1471-2334-5-24.

6. Alouane T, et al. First whole-genome sequences of two multidrug-resistant Acinetobacter baumannii strains isolated from a Moroccan hospital floor. Genome Announc. 2017;5(18):e00298-17.

7. Bergogne-Berezin $E$. The increasing role of Acinetobacter species as nosocomial pathogens. Curr Infect Dis Rep. 2001:3(5):440-4

8. Sun L, Wang X, Li Z. Successful treatment of multidrug-resistant Acinetobacter baumannii meningitis with ampicillin sulbactam in primary hospital. Br J Neurosurg. 2017:1-4. https://doi.org/10.1080/02688697.2017. 1319907.

9. Karaiskos I, et al. Successful treatment of extensively drug-resistant Acinetobacter baumannii ventriculitis and meningitis with intraventricular colistin after application of a loading dose: a case series. Int J Antimicrob Agents. 2013;41(5):480-3.

10. Fishbain J, Peleg AY. Treatment of Acinetobacter infections. Clin Infect Dis. 2010;51(1):79-84.

11. Jimenez $M$, et al. Treatment of multidrug-resistant Acinetobacter baumanni meningitis with ampicillin/sulbactam. Clin Infect Dis. 1997;24(5):932-5.

12. Guardado A, Blanco A, Cartón J. Ampicillin/sulbactam in combination: a review of its use in the treatment of severe bacterial infections. Clin Med Rev Ther. 2010;2010(2):245-57.

13. Peleg AY, Seifert H, Paterson DL. Acinetobacter baumannii: emergence of a successful pathogen. Clin Microbiol Rev. 2008;21(3):538-82.

14. Tunkel AR, Hasbun R, Bhimraj A, Byers K, Kaplan SL, Scheld WM, et al. Infectious Diseases Society of America's clinical practice guidelines for healthcareassociated ventriculitis and meningitis. Clin Infect Dis. 2017;64(6):34-65. 